\title{
Analysis of cross sections on iron and oxygen using Cf-252 neutron source
}

\author{
Bohumil Jansky ${ }^{1, *}$, Jiri Rejchrt ${ }^{1}$, Evzen Novak $^{1}$, and Anatoly Blokhin ${ }^{2}$ \\ ${ }^{1}$ Research Centre Rez, Hlavni 130, Husinec-Rez, Czech Republic \\ ${ }^{2}$ Nuclear Safety Institute of Russian Academy of Science, Moscow, Russia
}

\begin{abstract}
The leakage neutron spectra measurements have been done on benchmark spherical assemblies with Cf-252 source in center of 1) heavy water sphere with diameter of $30 \mathrm{~cm}$ (with Cd cover) and of 2) iron spheres with diameter of $100 \mathrm{~cm}$ and $50 \mathrm{~cm}$. It has been stated for years that transport calculations by iron overestimate measured spectra in energy region around $300 \mathrm{keV}$ by about $20-40 \%$ (calculation to measurement ratio $\mathrm{C} / \mathrm{E}=$ 1.2-1.4). The influence of an artificial changes in cross-section XS-Fe-56 (n,elastic)designed by IAEA, Nuclear Data Section, has been studied on the iron spheres. Influence of those XS-corrections to calculated neutron spectrum is presented.
\end{abstract}

\section{Introduction}

Validation of ${ }^{56} \mathrm{Fe}$ and ${ }^{16} \mathrm{O}$ cross section has been performed in Research Centre Rez (RC) in accordance with CIELO [1] project requirements. It has been stated for years that calculations for iron overestimate measured spectra in energy region around $300 \mathrm{keV}$ by about $20-40$ $\%$, i.e., determination of calculation to experiment ratio $\mathrm{C} / \mathrm{E}=1.2-1.4$ and also around $600 \mathrm{keV}$ by about $12-15$ $\%$. The $\mathrm{C} / \mathrm{E}$ around $300 \mathrm{keV}$ grows with iron thickness, B. Jansky [2], [3]. R. Capote, A. Trkov and S. Simakov from IAEA-NDS changed cross-section of ${ }^{56} \mathrm{Fe}$ producing new ND library IND-R22 and IND-R34 (IND - INDEN conference 2018, [4]) to test influence of such change.

\section{Experimental and calculation methods}

\subsection{Experimental setup}

Determination of $\mathrm{C} / \mathrm{E}$ ratio of leakage neutron spectra from iron and heavy water spheres with Cf-252 neutron source in center was done for 4 libraries IND-R22, INDR34, CIELO and JEFF $3.2 \mathrm{~T} 2$ for ${ }^{56} \mathrm{Fe}$ and CIELO for ${ }^{16} \mathrm{O}$. The following assemblies were used for measurement and calculation: 1) heavy water sphere with diameter of $30 \mathrm{~cm}$ (with $\mathrm{Cd}$ cover) and 2) iron spheres with diameter of $100 \mathrm{~cm}$ and $50 \mathrm{~cm}$. Each assembly had certain acronym, e.g., FE100R150 is Fe assembly with the diameter of $100 \mathrm{~cm}$ and the distance of $150 \mathrm{~cm}$ between sphere and detector (center to center). At first, the leakage neutron (and gamma) spectrum from spherical assemblies with Cf-252 in center was measured together with background. Then, the background itself was measured with shielding cone placed between the sphere and detector. To get "pure" leakage spectrum, the measured background was subtracted from the first measurement.

*e-mail: bohumil.jansky@cvrez.cz

\subsection{Calculation}

The calculations were performed using Monte-Carlo code MCNP with nuclear data library CIELO (i.e., ENDF/BVIII.0) and JEFF-3.2T2. As for calculation geometry description, a simplified model was used, which substitutes assembly elements with concentric spherical shells around the source. Also, the MCNP model of detector is represented by a $1 \mathrm{~cm}$ thick spherical shell with radius equal to the real distance $\mathrm{R}$ of detector and source. For each calculation $10^{8}-10^{9}$ particle histories were computed.

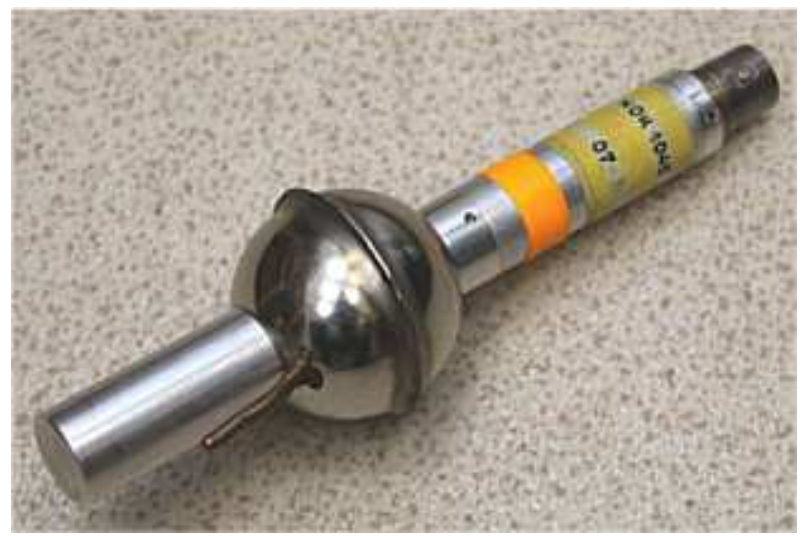

Figure 1. Hydrogen proportional detector of NOK-445 type.

\subsection{Spectrometry}

Two hydrogen proportional spherical detectors (HPD) K4 and $\mathrm{K} 8$ with diameter of $40 \mathrm{~mm}$ were used in neutron spectrometer [5]. The detector $\mathrm{K} 4$ with pressure $400 \mathrm{kPa}$ was used for measurement in the energy range $E_{n}=0.01-0.7$ $\mathrm{MeV}$, the detector $\mathrm{K} 8$ with pressure $1000 \mathrm{kPa}$ was used for measurement in the energy range $E_{n}=0.2-1.3 \mathrm{MeV}$, see 
Figure 1. Stilbene spectrometer was used for measurement in the range $E_{n}=1-10 \mathrm{MeV}$. The HPD measured and calculated spectra were evaluated in two group structures: 40 gpd, it corresponds to the lethargy step about $6 \%$ and in structure $200 \mathrm{gpd}$, i.e., with lethargy step $1 \%$. The common energy structure for stilbene had step of $100 \mathrm{keV}$.

\subsection{Uncertainties}

Uncertainty of single measurement is composed of uncertainty of the "A-type" that includes statistical uncertainty in measurement (in channel) and consequent calculation of each energy group and uncertainty of "B-type" that includes influence of apparatus instability, of benchmark geometry and detector position, neutron source position, detector discharges, energy calibration, during time remote repeated measurements. Uncertainties of A-type of the integral values presented in tables 1-3 are in interval from 0.3 to $5 \%$. Uncertainty of "B-type" we estimate $2-4 \%$. Uncertainties of MCNP calculations are better than $1 \%$ in energy interval $0.1-1.3 \mathrm{MeV}$ and better than $5 \%$ in energy interval $1-5 \mathrm{MeV},[6]$.

\begin{tabular}{|c|c|c|c|c|c|c|c|}
\hline \multicolumn{2}{|c|}{$E_{n}[\mathrm{MeV}]$} & \multicolumn{6}{|c|}{ Integral values [1] } \\
\hline From & To & Measurement & Unc [\%] & CIELO & Unc [\%] & $\mathrm{C} / \mathrm{E}$ & Unc [\%] \\
\hline 0.10 & 10.00 & 0.28930 & 0.57 & 0.30410 & 0.04 & 1.051 & 0.57 \\
\hline 0.10 & 0.20 & 0.04543 & 1.84 & 0.04818 & 0.10 & 1.061 & 1.85 \\
\hline 0.20 & 0.30 & 0.02343 & 1.96 & 0.02510 & 0.14 & 1.071 & 1.96 \\
\hline 0.30 & 0.50 & 0.02258 & 2.72 & 0.02315 & 0.15 & 1.025 & 2.73 \\
\hline 0.50 & 1.00 & 0.03836 & 2.70 & 0.04197 & 0.11 & 1.094 & 2.71 \\
\hline 1.00 & 2.00 & 0.05331 & 0.99 & 0.05536 & 0.09 & 1.038 & 0.99 \\
\hline 2.00 & 3.00 & 0.04819 & 0.23 & 0.04884 & 0.10 & 1.013 & 0.25 \\
\hline 3.00 & 5.00 & 0.03971 & 0.32 & 0.04154 & 0.11 & 1.046 & 0.34 \\
\hline 5.00 & 7.00 & 0.01409 & 0.77 & 0.01499 & 0.18 & 1.064 & 0.79 \\
\hline \multirow[t]{2}{*}{7.00} & 10.00 & 0.00422 & 1.81 & 0.00497 & 0.31 & 1.179 & 1.84 \\
\hline & & D abs $<5 \%$ & $D=5-10 \%$ & $\mathrm{D}>10 \%$ & $D=-(5-10 \%)$ & $D<-10 \%$ & \\
\hline
\end{tabular}

Figure 2. Comparison of calculated and measured spectra D2O30R100, E: HPD+stilbene, C: CIELO.

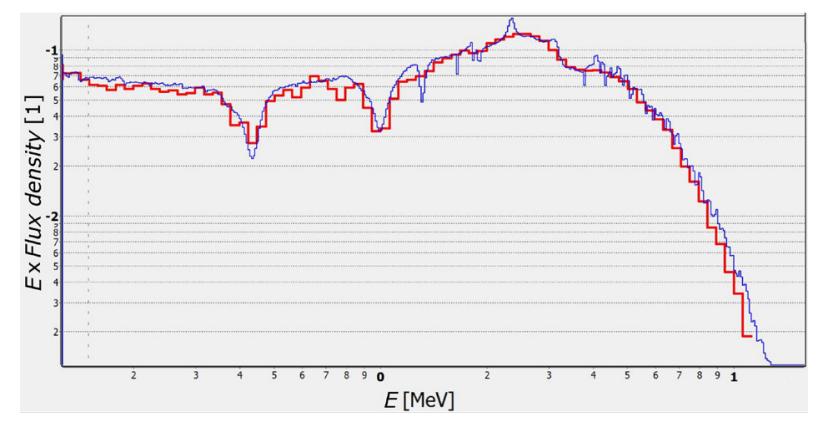

Figure 3. Comparison of calculated and measured spectra - Assembly D2O30R100, E: HPD+stilbene(red), C: CIELO (blue).

\section{Validation of ${ }^{16} \mathrm{O}$ cross section}

Validation of ${ }^{16} \mathrm{O}$ cross section was performed on D2O30R100 assembly. Neutron spectrum was measured by 2 HPDs K4 and K8 (Research Centre Rez) and stilbene
(Military Academy Brno, F. Cvachovec, [6]). The CIELO (ENDF/B-VIII.0) was used for calculation. Results of C/E are in Figures 2 (table) and 3 (graph).

\section{Analysis of ${ }^{56} \mathrm{Fe}(\mathrm{n}, \mathrm{el})$ cross section}

\subsection{Energy region $300 \mathrm{keV}$}

Results of validation of 4 different ND libraries including corrected ND IND-R22 and IND-R34 in the energy region $E_{n}<1 \mathrm{MeV}$ are in Figure 4 (table) and Figure 5 (graph), [2], [3], [8].

\begin{tabular}{cccccc}
\hline \multicolumn{2}{c}{$\boldsymbol{E}_{\boldsymbol{n}}[\mathrm{MeV}]$} & \multicolumn{4}{c}{ C/E ratio } \\
\hline From & To & CIELO & JEFF-3.2T2 & IND-R22 & IND-R34 \\
\hline $\mathbf{0 . 0 1 3}$ & $\mathbf{1 . 2 9 0}$ & $\mathbf{1 . 0 4 5 0}$ & $\mathbf{1 . 0 5 2 0}$ & $\mathbf{1 . 0 4 6 0}$ & $\mathbf{1 . 0 2 9 0}$ \\
\hline 0.013 & 0.033 & 0.9138 & 0.9958 & 0.9657 & 1.0560 \\
0.033 & 0.060 & 0.9005 & 1.0190 & 0.9890 & 1.0940 \\
0.060 & 0.090 & 0.9702 & 0.9894 & 1.0720 & 1.1290 \\
0.090 & 0.150 & 0.9934 & 1.0070 & 1.1150 & 1.0130 \\
0.150 & 0.200 & 1.0370 & 1.0070 & 1.1790 & 1.0900 \\
0.200 & 0.250 & 1.0280 & 1.0130 & 1.1670 & 1.0390 \\
0.250 & 0.289 & 1.0360 & 1.0050 & 0.9749 & 0.9561 \\
0.289 & 0.333 & 1.3330 & 1.2290 & 1.0140 & 0.9150 \\
0.333 & 0.367 & 1.3050 & 1.2680 & 1.0640 & 0.9819 \\
0.367 & 0.410 & 1.1910 & 1.1710 & 0.8036 & 1.0450 \\
0.410 & 0.520 & 1.0330 & 1.0810 & 0.9663 & 1.0070 \\
0.520 & 0.780 & 1.0890 & 1.0620 & 1.0430 & 1.0440 \\
0.780 & 1.060 & 0.7834 & 1.0490 & 0.9974 & 0.9984 \\
1.060 & 1.290 & $\mathbf{0 . 7 5 8 4}$ & 0.8654 & 1.0510 & 1.0530 \\
& & & & & \\
& & D abs<5\% & D=5-10\% & D>10\% & D=-(5-10\%) \\
& & D<-10\% & & &
\end{tabular}

Figure 4. Comparison of calculated and measured spectra - assembly FE100R53,“ HPD region”, E: HPD, C: CIELO, JEFF, IND-R22 and IND-R34.

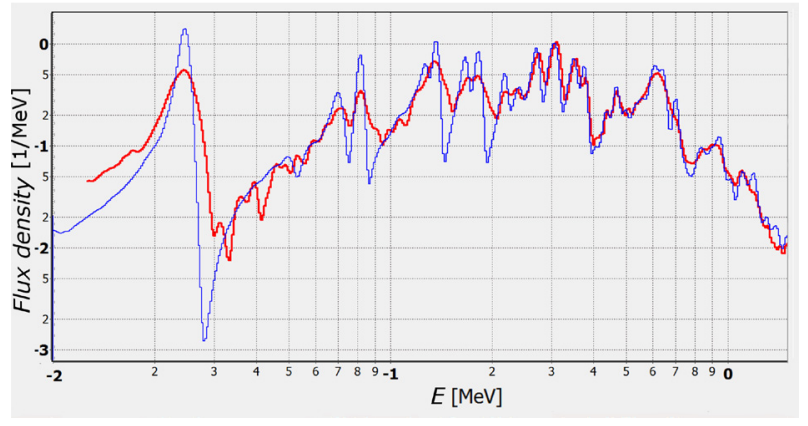

Figure 5. Comparison of calculated and measured spectra - assembly FE100R53,“ HPD region”, E: HPD (red), C: IND-R34 (blue), 200 gpd.

\subsection{Comparison of ND libraries on two Iron Benchmarks}

The measurements were performed on iron spheres with diameter of $50 \mathrm{~cm}$ (FE50) and of $100 \mathrm{~cm}$ (FE100). Two type of spectrometry results were used for cross section 
validation:

1) FE100R53, $E_{n}=0.013-1.3 \mathrm{MeV}$, "HPD"region"

2) FE50R100, $E_{n}=0.8-17 \mathrm{MeV}$, "stilbene region"

The spectra in the stilbene region were averaged from 4 independent measurements (L.A. Trykov [3], F. Cvachovec [6], M. Košt'ál - stilbene type and J. Adams "ROSPEC" type-HPD [9]) to minimize uncertainty of "B-type" in individual authors methodology of measurement, gamma discrimination, evaluation, Cf neutron source properties description, see [8], [9]. The cross section of ${ }^{56} \mathrm{Fe}(\mathrm{n}, \mathrm{el})$ isotope is dominant iron reaction in energy region $E_{n}<0.7 \mathrm{MeV}$. Moreover ${ }^{56} \mathrm{Fe}$ isotope has $92 \%$ share in iron. Results of IND-R22 and IND-R34 with corrected ${ }^{56} \mathrm{Fe}(\mathrm{n}, \mathrm{el})$ cross section validation and also of CIELO and JEFF-3.2T2 are in the Figures 4 (table), Figure 5 (graph) and Figures 6 (table), Figure 7 (graph).

\subsection{Influence of spectrometer resolution on spectra assessment}

Measurements and calculations use 200 gpd energy structure, i.e., lethargy step is about $1 \%$. Spectrometer with HPD has relatively good resolution, because in the energy interval 200-400 keV 4-6 peaks are visible: $218,242,272$, 309, 352 and $375 \mathrm{keV}$. See Figure 5. Other spectrometers that use stilbene or TOF method resolve usually only one "thick" peak at $300 \mathrm{keV}$ in the region 200-400 keV. Results are in Figure 4 and Figure 5. Correction of ${ }^{56} \mathrm{Fe}$ (n,tot) cross section is in the Figure 8, where CIELO is compared with IND-R34 data version.

\begin{tabular}{cccccc}
\hline \multicolumn{2}{c}{$\boldsymbol{E} \boldsymbol{n}[\mathrm{MeV}]$} & \multicolumn{5}{c}{ C/E ratio } \\
\hline From & To & CIELO & JEFF-3.2T 2 & IND-R22 & IND-R34 \\
\hline $\mathbf{1 . 0}$ & $\mathbf{1 0 . 0}$ & $\mathbf{0 . 8 5 9}$ & $\mathbf{0 . 9 9 2}$ & $\mathbf{1 . 0 1 5}$ & $\mathbf{1 . 0 1 5}$ \\
\hline 0.8 & 0.9 & 0.921 & 1.120 & 0.979 & 0.979 \\
0.9 & 1.0 & 0.957 & 1.080 & 1.038 & 1.038 \\
1.0 & 1.2 & 0.874 & 0.941 & 0.967 & 0.967 \\
1.2 & 1.4 & 0.868 & 0.971 & 1.034 & 1.034 \\
1.4 & 1.6 & 0.773 & 0.901 & 0.971 & 0.971 \\
1.6 & 1.8 & 0.893 & 1.032 & 1.083 & 1.083 \\
1.8 & 2.0 & 0.913 & 1.095 & 1.116 & 1.116 \\
2.0 & 3.0 & 0.852 & 1.114 & 1.044 & 1.044 \\
3.0 & 4.0 & 0.817 & 1.193 & 1.089 & 1.089 \\
4.0 & 5.0 & 0.843 & 1.120 & 1.064 & 1.064 \\
5.0 & 6.0 & 0.857 & 1.022 & 1.035 & 1.035 \\
6.0 & 7.0 & 0.874 & 1.029 & 1.020 & 1.020 \\
7.0 & 8.0 & 0.878 & 1.040 & 1.013 & 1.013 \\
8.0 & 9.0 & 0.940 & 1.110 & 1.083 & 1.083 \\
9.0 & 10.0 & 0.912 & 1.058 & 1.041 & 1.041 \\
10.0 & 12.0 & 0.895 & 0.975 & 0.983 & 0.983 \\
12.0 & 14.0 & 0.799 & 0.803 & 0.824 & 0.824 \\
14.0 & 16.0 & 0.672 & 0.638 & 0.673 & 0.673 \\
& & & & & \\
& & $\mathbf{D}$ abs<5\% & $\mathbf{D}=\mathbf{5 - 1 0 \%}$ & $\mathbf{D}>\mathbf{1 0} \%$ & $\mathbf{D}=-\mathbf{( 5 - 1 0 \% )}$ \\
& & $\mathbf{D}<-\mathbf{1 0} \%$ & & &
\end{tabular}

Figure 6. Comparison of calculated and measured spectra - assembly FE50R100,"stilbene region", E: averaged from 4 measurement, C: CIELO, JEFF-3.2T2, IND-R22, IND-R34.

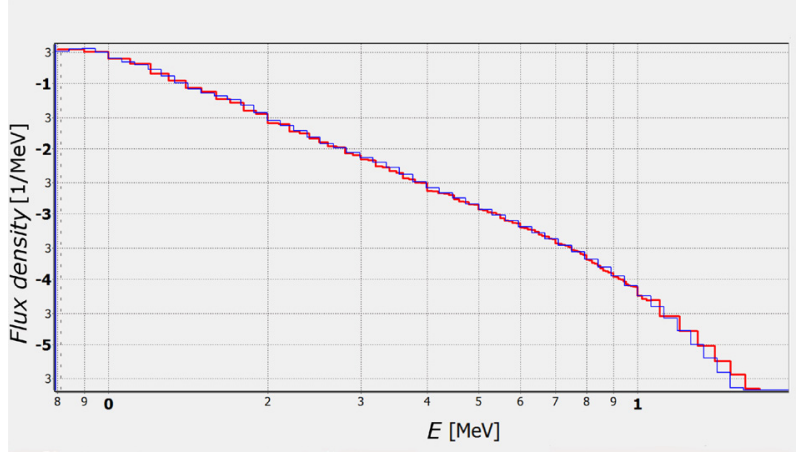

Figure 7. Comparison of calculated and measured spectraFE50R100,“stilbene region”, E: red line, C: IND-R34, blue line.

\section{Conclusion}

Validation of ${ }^{16} \mathrm{O}$ cross section - Figure 2 (table) and Figure 3 (graph), assembly D2O30R100, C/E = 1.02$1.09, \mathrm{C} / \mathrm{E}=1.18$ for $7-10 \mathrm{MeV}$ ) - proves that calculation systematically slightly overestimates the measurement.

Analysis of ${ }^{56} \mathrm{Fe}(\mathrm{n}, \mathrm{el})$ cross section - Figure 4 (table) and Figure 5 (graph), IAEA corrections for $E_{n}=$ 0.013-0.7 MeV, "HPD region" - shows that changes of Fe cross section in IND-R34 ND bring better agreement in $\mathrm{C} / \mathrm{E}$, but changes affect other surrounding energy regions. Undesirable changes of $\mathrm{C} / \mathrm{E}$ ratio for correction performed in IND-R34 opposite to IND-R22 are in following energy intervals $\left(E_{n}\right.$ is in $\left.\mathrm{MeV}\right)$ :

$E_{n}=0.033-0.060: \mathrm{C} / \mathrm{E}$ increases from 0.989 to 1.094

$E_{n}=0.060-0.090: \mathrm{C} / \mathrm{E}$ increases from 1.072 to 1.129

$E_{n}=0.289-0.333: \mathrm{C} / \mathrm{E}$ decreases from 1.014 to 0.915

Analysis of ${ }^{56} \mathrm{Fe}(\mathrm{n}, \mathrm{el})$ cross section - Figure 6 (table), energy region $E_{n}=0.8-16 \mathrm{MeV}$, "stilbene region" proves that for CIELO all values of $\mathrm{C} / \mathrm{E}$ are very low, i.e, calculation underestimates measurements systematically by $5-20 \%$. Calculation with data versions IND-R22 and IND-R34 exhibits better values than CIELO and JEFF in mentioned region. The ratio $\mathrm{C} / \mathrm{E}=1.08-1.12$ for both IND-R22 and IND-R34 libraries is worse in energy region $E_{n}=1.6-2 \mathrm{MeV}$ than in the other.

It seems to be important to reassess the creation of the ${ }^{56} \mathrm{Fe}(\mathrm{n}, \mathrm{el})$ cross section, e.g. M. Diakaki, CEA Cadarache, [10].

\section{Acknowledgments}

Presented results were obtained with the use of infrastructure Reactors LVR-15 and LR-0, which is financially supported by the Ministry of Education, Youth and Sports project LM2015074 and also financially supported by the Ministry of Education, Youth and Sports Czech Republic - project LQ1603 Research for SUSEN. 


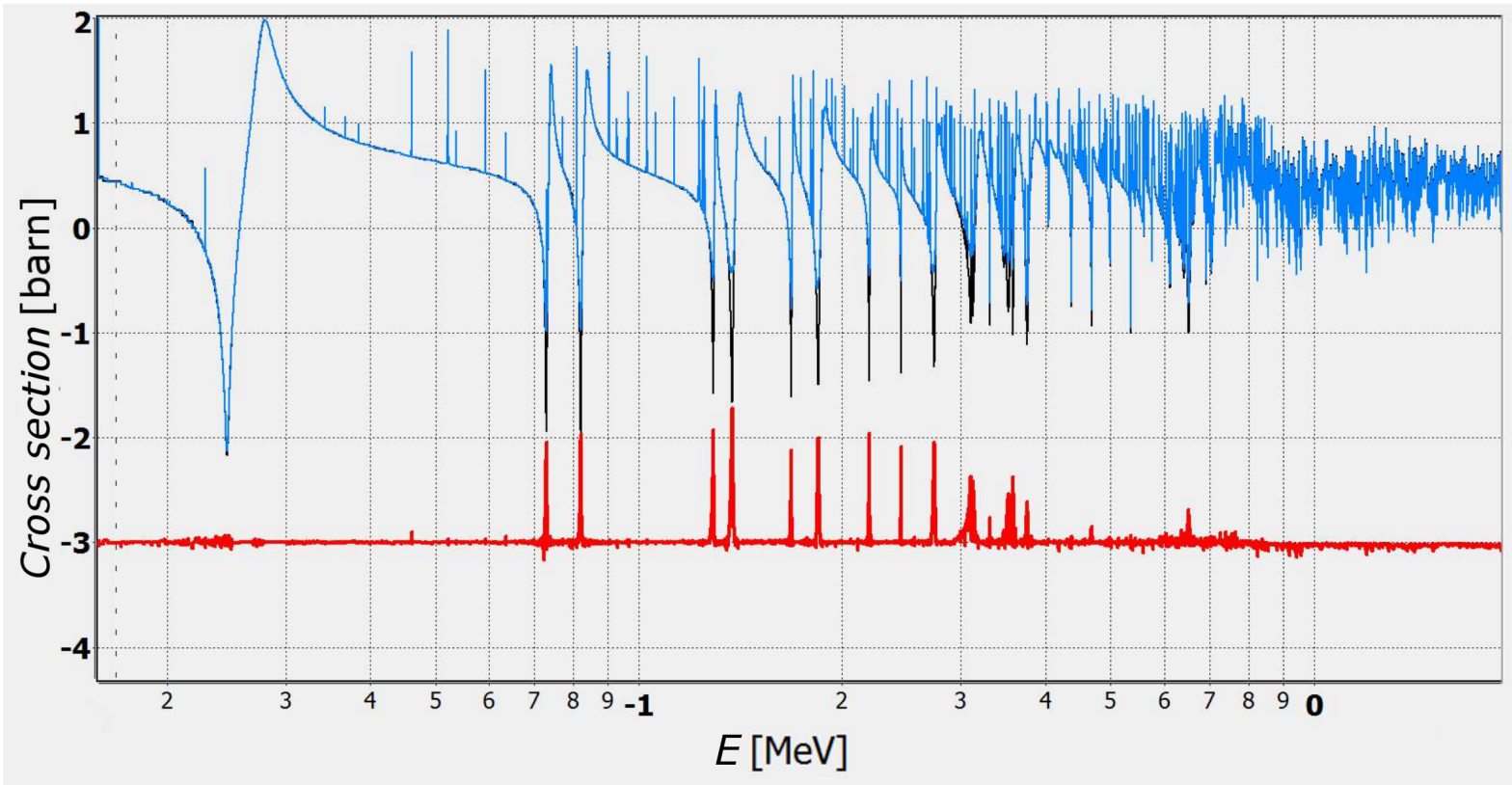

Figure 8. Cross section of ${ }^{56} \mathrm{Fe}$ (n,tot) comparison: CIELO (black), R34 (blue), R34/CIELO - norm = 1000 (red).

\section{References}

[1] M.B. Chadwick, CIELO: A Future Collaborative International Evaluated Library, conference Nuclear Data, ND 2013, New York, 2013.

[2] B. Jansky, J. Rejchrt, M. Schulc, A. Blokhin, "Iron-56, problem with the elastic cross section in neutron energy region around $300 \mathrm{keV}$ and natural iron isotopes influence on the neutron transport through iron." JEFDOC1918, NEA Nuclear Data Week - JEFF Meetings, 18 - 20 April 2018, CIEMAT, Moncloa Centre, Madrid, Spain.

[3] B. Jansky, J. Rejchrt, E. Novak, E. Losa, A. I. Blokhin and E. Mitenkova, Neutron Spectra Measurement and Calculations using Data Libraries CIELO, JEFF-3.2 and ENDF/B-VII.1 in Iron Benchmark Assemblies, Nuclear Data 2016, Bruges, Belgium, 11-16.09.2016, (contribution No. R152).

[4] A. Trkov, R. Capote, On the Benchmarking of INDEN Improved Iron Evaluations (INDC(NDS)-0757). International Atomic Energy Agency (IAEA), (2018).

[5] B. Jansky, E. Novak, Neutron Spectrometry with Spherical Hydrogen Proportional Detectors, Nuclear Instruments and Methods in Physics Research, A735(2014), 390-398.
[6] B. Jansky, Z. Turzik, E. Novak, J. Kyncl, F. Cvachovec, P. Tiller.: Comparison of measured and calculated Neutron transmission through heavy water for 252 Cf source placed in the center of $30 \mathrm{~cm}$ diameter sphere, Annals of Nucl. Energy, Vol. 24, No. 15, pp 1189-1212, 1997.

[7] B. Jansky, E. Novak , P. Otopal .: Data for calculation of neutron and gamma leakage spectra from iron and water spheres with Cf-252 neutron source in centre, Report NRI, ÚJV-11506, Řež, 2000.

[8] B. Jansky J.Rejchrt, A .Blokhin Neutron spectra measurement and calculation using data libraries JEFF-3.1, JEFF-3.2, JEFF-3.3 and ENDF/B-VIII.0 (CIELO) in Iron benchmark assemblies of RC Rez Laboratory report No. 201906 17, version-1; background material for JEFF-3.3 paper, edited by A. Plompen), Rez June 2019.

[9] M.B. Stanka, J.M. Adams, Ch.M. Eisenhauer "Proton Recoil Measurements of 252-Cf fission Neutron Leakage Spectrum from an Iron Sphere“, Nuclear Science and Engineering: 134, 68-76 (2000).

[10] M. Diakaki, S. Shen, G. Noguere, D. Bernard, P. Tamagno, P. Archier, CEA Cadarache,Evaluation of neutron induced reactions on $56 \mathrm{Fe}$ with CONRAD, ND 2019, Beijing. 